\title{
The Negishi Catalysis: Full Study of the Complications in the Transmetalation Step and Consequences for the Coupling Products
}

Juan del Pozo, ${ }^{1}$ Gorka Salas, ${ }^{2}$ Rosana Álvarez, ${ }^{* 3}$ Juan A. Casares, ${ }^{* 1}$ and Pablo Espinet ${ }^{* 1}$

${ }^{1}$ IU CINQUIMA/Química Inorgánica, Facultad de Ciencias, Universidad de Valladolid, 47011-Valladolid (Spain)

${ }^{2}$ IMDEA Nanociencia, Ciudad Universitaria de Cantoblanco, 28049 Madrid, Spain.

${ }^{3}$ Departamento de Química Orgánica, Facultad de Química (CINBIO), Universidade de Vigo. Campus As LagoasMarcosende, 36310 Vigo (Spain)

Index

General methods......................................................... S2

Synthesis of the complexes $\quad$ S2

Kinetic experiments $\quad$ S2

Data input for non-linear leas square fitting $\quad$ S9

$\begin{array}{ll}\text { Computational details } & \text { S15 }\end{array}$

Structures of the calculated complexes and transition states $\quad$ S16 


\section{General Methods}

All reactions were carried out under $\mathrm{N}_{2}$ or Ar in THF dried using a Solvent Purification System (SPS). NMR spectra were recorded on Bruker ARX 300, AV 400 or AV 500 instruments equipped with variable-temperature probes. Chemical shifts are reported in ppm from tetramethylsilane $\left({ }^{1} \mathrm{H}\right)$, and $\mathrm{CCl}_{3} \mathrm{~F}\left({ }^{19} \mathrm{~F}\right)$, with positive shifts downfield, at ambient probe temperature unless otherwise stated. The temperature for the NMR probe was calibrated with an ethylene glycol standard (high temperature) and with a methanol standard (low temperature). ${ }^{1}$ In the ${ }^{19} \mathrm{~F}$ and ${ }^{31} \mathrm{P}$ NMR spectra registered in non-deuterated solvents, a coaxial tube containing acetone- $\mathrm{d}_{6}$ was used to maintain the lock ${ }^{2} \mathrm{H}$ signal, and the chemical shifts are reported from the $\mathrm{CCl}_{3} \mathrm{~F}$ signal in deuterated acetone. The compounds trans-[PdRfMe $\left.\left(\mathrm{PPh}_{3}\right)_{2}\right](\mathbf{1})$, trans$\left[\mathrm{PdRfMe}\left(\mathrm{PPh}_{3}\right)_{2}\right](2)$ and $c$ is-[PdRfMe $\left.\left(\mathrm{PPh}_{3}\right)_{2}\right](3)$ were prepared as reported in the literature. ${ }^{2}$

\section{Synthesis of the complexes}

trans-[PdMe $\left.\mathbf{P}_{2}\left(\mathbf{P P h}_{3}\right)_{2}\right]$. This is a modification of the published procedure. ${ }^{3}$ Trans$\left[\mathrm{PdClMe}\left(\mathrm{PPh}_{3}\right)_{2}\right](200 \mathrm{mg}, 0.30 \mathrm{mmol})$ and $\mathrm{PPh}_{3}(77.6 \mathrm{mg}, 0.30 \mathrm{mmol})$ were dissolved in 30 $\mathrm{mL}$ of THF in a two necked flask and stirred until all the solid was totally dissolved. The solution was cooled to $\left(-80^{\circ} \mathrm{C}\right)$, and then, $\mathrm{ZnMe}_{2}(1.5 \mathrm{~mL}, 1.5 \mathrm{mmol}, 2 \mathrm{M}$ in toluene $)$ was added dropwise. When the addition was finished, extra $\mathrm{ZnMe}_{2}$ was added fastly $(1.5 \mathrm{~mL}, 1.5 \mathrm{mmol}$, $2 \mathrm{M}$ in toluene). The reaction was stirred at $\left(-80^{\circ} \mathrm{C}\right)$ for two hours. Precooled hexane at $\left(-80^{\circ} \mathrm{C}\right)$ was added to force a white solid to precipitate. It was filtered and washed with hexane $(2 \mathrm{x}$ $5 \mathrm{~mL})$ and dried under reduced pressure. Yield $140 \mathrm{mg}(71 \%)$. The solid must be checked at $210 \mathrm{~K}$ in order to keep the complex away from isomerization. The sample must be carried out in the presence of added $\mathrm{PPh}_{3}$ (1 equivalent) or cis-[ $\left.\mathrm{PdMe}_{2}\left(\mathrm{PPh}_{3}\right)_{2}\right]$ would be formed. ${ }^{1} \mathrm{H}$ NMR (400 MHz, THF-d8, 210K) $\delta 7.71(\mathrm{q}, J=5.8 \mathrm{~Hz}, 2 \mathrm{H}), 7.62-7.48(\mathrm{~m}, 13 \mathrm{H}), 7.41(\mathrm{~m}, 21 \mathrm{H}),-$ $0.89(\mathrm{t}, J=5.4 \mathrm{~Hz}, 6 \mathrm{H}) .{ }^{31} \mathrm{P}\left\{{ }^{1} \mathrm{H}\right) \mathrm{NMR}(162 \mathrm{MHz}, \mathrm{THF}-\mathrm{d} 8,210 \mathrm{~K}) \delta 42.84$ (s). Anal. Calcd for $\mathrm{C}_{38} \mathrm{H}_{36} \mathrm{P} 2 \mathrm{Pd}$ : C, 69.04; H, 5.49. Found: C, 68.79; H 5.32.

\section{Kinetic experiments}

In a standard experiment a solution of palladium complex trans-[PdRfCl$\left.\left(\mathrm{PPh}_{3}\right)_{2}\right]$ (1) trans[PdRfMe $\left.\left(\mathrm{PPh}_{3}\right)_{2}\right](2)$ or cis-[PdRfMe $\left.\left(\mathrm{PPh}_{3}\right)_{2}\right](3)\left(10 \mathrm{mg}, 1.13 \times 10^{-2} \mathrm{mmol}\right)$ and $\mathrm{PPh}_{3}(0$ to 6 $\mathrm{mg} ; 0$ to $\left.2.3 \times 10^{-2} \mathrm{mmol}\right)$ in THF $(0.40 \mathrm{~mL})$ was prepared in a RMN tube and cooled to $-80{ }^{\circ} \mathrm{C}$. A solution of $\mathrm{ZnMe}_{2} 2 \mathrm{M}$ in toluene $(0.20 \mathrm{~mL}, 0.40 \mathrm{mmol})$ was added plus cold THF to make $0.60 \mathrm{~mL}$ of final volume. Then a coaxial capillary containing acetone- $d_{6}$ was added, and the 
sample was placed into the NMR probe thermostated at $25^{\circ} \mathrm{C}$. The kinetic experiments were followed by ${ }^{31} \mathrm{P}$ NMR or ${ }^{19} \mathrm{~F}$ NMR and Temperature equilibration of the sample was reached after 5 minutes inside the NMR probe. Evolution of the reaction was monitored by ${ }^{31} \mathrm{P}$ NMR or by ${ }^{19} \mathrm{~F}$ NMR spectroscopy. Concentration-time data were acquired by integration of the ${ }^{19} \mathrm{~F}$ NMR signals.

Transmetalation studies in the presence of added $P P h_{3}$.

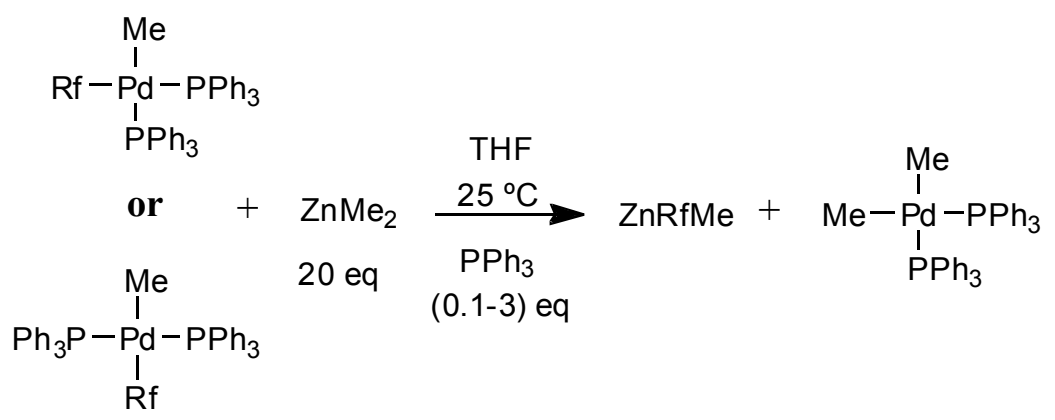

Scheme S1. Transmetalation reaction between trans-[PdRfMe $\left.\left(\mathrm{PPh}_{3}\right)_{2}\right]$ or cis-[PdRfMe $\left.\left(\mathrm{PPh}_{3}\right)_{2}\right]$ with different $\left[\mathrm{PPh}_{3}\right]$.

Table S1. Experimental values for transmetalation kinetic studies. Complex cis-[PdRfMeL 2$]$ (1a).

\begin{tabular}{ccccc}
\hline$\left[\mathrm{PPh}_{3}\right] \mathrm{M}$ & $-\mathrm{r}_{0}\left(\mathrm{M} \cdot \mathrm{s}^{-1}\right)$ & cis- $\left[\mathrm{PdRfMeL}_{2}\right]$ & {$\left[\mathrm{ZnMe}_{2}\right]$} & $\mathrm{k} \mathrm{obs}$ \\
\hline $16.2 \cdot 10^{-3}$ & $3.62 \cdot 10^{-8}$ & 0.0197 & 0.6667 & $2.76 \cdot 10^{-6}$ \\
$13.1 \cdot 10^{-3}$ & $4.63 \cdot 10^{-8}$ & 0.0197 & 0.6667 & $3.53 \cdot 10^{-6}$ \\
$7.3 \cdot 10^{-3}$ & $6.38 \cdot 10^{-8}$ & 0.0197 & 0.6667 & $4.86 \cdot 10^{-6}$ \\
$4.8 \cdot 10^{-3}$ & $1.00 \cdot 10^{-7}$ & 0.0195 & 0.6667 & $7.70 \cdot 10^{-6}$ \\
$3.3 \cdot 10^{-3}$ & $1.82 \cdot 10^{-7}$ & 0.0198 & 0.6667 & $1.38 \cdot 10^{-5}$ \\
$1.4 \cdot 10^{-3}$ & $3.23 \cdot 10^{-7}$ & 0.0196 & 0.6667 & $2.47 \cdot 10^{-5}$
\end{tabular}

Table S2. Experimental values for transmetalation kinetic studies. Complex trans-[PdRfMeL 2$](\mathbf{2 a})$.

\begin{tabular}{ccccc}
\hline$\left[\mathrm{PPh}_{3}\right] \mathrm{M}$ & $\begin{array}{c}-\mathrm{r}_{0}\left(\mathrm{M} \cdot \mathrm{s}^{-}\right. \\
1)\end{array}$ & $\begin{array}{c}\text { trans }- \\
{\left[\mathrm{PdRfMeL}_{2}\right]}\end{array}$ & {$\left[\mathrm{ZnMe}_{2}\right]$} & $\mathrm{k} \mathrm{obs}$ \\
\hline $16.7 \cdot 10^{-3}$ & $2.26 \cdot 10^{-7}$ & 0.0197 & 0.6667 & $1.72 \cdot 10^{-5}$ \\
$6.2 \cdot 10^{-3}$ & $3.29 \cdot 10^{-7}$ & 0.0196 & 0.6667 & $2.51 \cdot 10^{-5}$ \\
$3.3 \cdot 10^{-3}$ & $4.14 \cdot 10^{-7}$ & 0.0197 & 0.6667 & $3.16 \cdot 10^{-5}$ \\
$1.5 \cdot 10^{-3}$ & $5.53 \cdot 10^{-7}$ & 0.0197 & 0.6667 & $4.20 \cdot 10^{-5}$ \\
$10.1 \cdot 10^{-3}$ & $2.92 \cdot 10^{-7}$ & 0.0196 & 0.6667 & $2.23 \cdot 10^{-5}$ \\
$138.6 \cdot 10^{-3}$ & $5.70 \cdot 10^{-8}$ & 0.0198 & 0.6667 & $4.33 \cdot 10^{-6}$
\end{tabular}




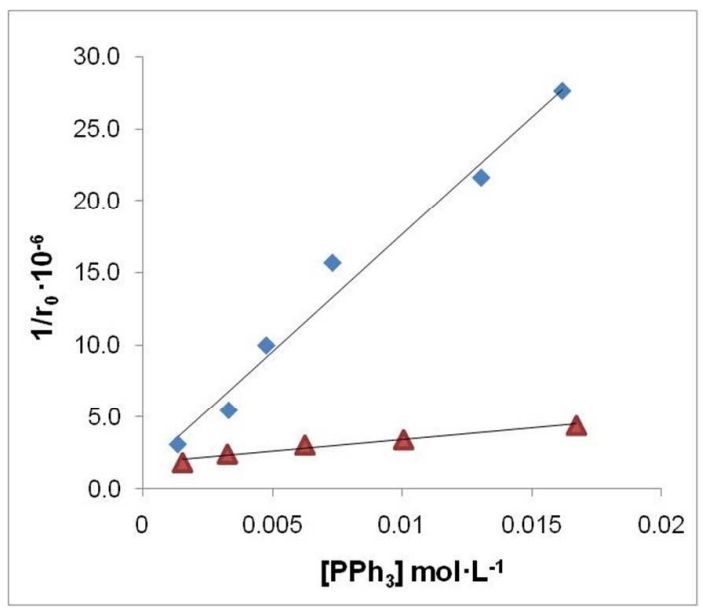

Figure S1. Plot of the reversal of the rate of formation of $\mathrm{ZnRfMe} v \mathrm{~s}$. [ $\left.\mathrm{PPh}_{3}\right]$ starting from trans-[PdRfMe $\left.\left(\mathbf{P P h}_{3}\right)_{2}\right]\left(\right.$ red spots, $\left.\mathrm{y}=1.6 \cdot 10^{8} \mathrm{x}+\mathbf{1 . 8} \cdot 10^{\mathbf{6}} ; \mathrm{R}^{2}=0.97\right)$ or $\operatorname{cis}-\left[\mathbf{P d R f M e}\left(\mathbf{P P h}_{3}\right)_{2}\right]$ (blue spots, $\mathrm{y}=1.6 \cdot 10^{9} \mathrm{x}+\mathbf{0 . 1} \cdot \mathbf{1 0} \mathbf{7}^{7} ; \mathrm{R}^{2}=0.98$ ).

Table S3. Confidence intervals of the linear regression.

\begin{tabular}{lll}
\hline & Slope & interception \\
\hline cis-[PdRfMe $\left.\left(\mathrm{PPh}_{3}\right)_{2}\right]$ & $\left(1.3 \cdot 10^{9}-1.9 \cdot 10^{9}\right)$ & $\left(-1.6 \cdot 10^{6}-4.5 \cdot 10^{6}\right)$ \\
trans-[PdRfMe $\left.\left(\mathrm{PPh}_{3}\right)_{2}\right]$ & $\left(1.1 \cdot 10^{8}-2.2 \cdot 10^{8}\right)$ & $\left(13 \cdot 10^{6}-23 \cdot 10^{6}\right)$
\end{tabular}

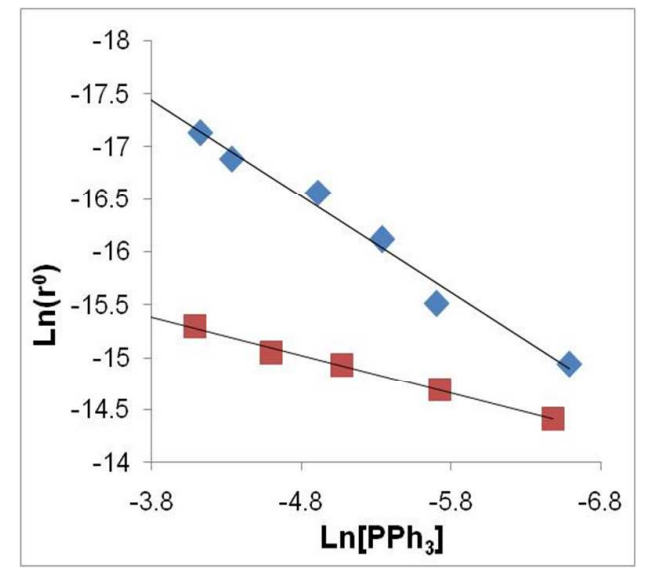

Figure S2. Representation of $\ln \left[\mathrm{r}^{0}\right]$ (formation of $\mathrm{ZnRfMe}$ ) $v s . \ln \left[\mathrm{PPh}_{3}\right]$ from (2a) (red spots, $\mathrm{y}$ $\left.=\mathbf{- 0 . 3 6 2} x-16.75 ; R^{2}=0.993\right)$ or $(\mathbf{1 a})\left(\right.$ blue spots, $\left.y=\mathbf{- 0 . 9 0 9 x}-20.89 ; R^{2}=0.980\right)$. The slope represents the kinetic order of the reactions on $\left[\mathrm{PPh}_{3}\right]$. 
Table S4.Confidence intervals of the linear regression.

\begin{tabular}{ccc}
\hline & Slope & interception \\
\hline cis-[PdRfMe $\left.\left(\mathrm{PPh}_{3}\right)_{2}\right]$ & $(-1.09)-(-0.73)$ & $(-21.83)-(-19.96)$ \\
trans-[PdRfMe $\left.\left(\mathrm{PPh}_{3}\right)_{2}\right]$ & $(-0.42)-(-0.31)$ & $(-17.04)-(-16.47)$
\end{tabular}

\section{Proposed models}

The fitting of rate constants was performed with the aid of the software "COPASI". ${ }^{\circ}$ Only data points (concentration vs. time of all the species) concerning up to $12 \%$ of conversion were used for the fitting. This allows considering the reaction as irreversible. At long reaction times, $\operatorname{Pd}(0)$ complexes are formed and behave as ligand scavengers and other decomposition products start to appear. Values of the concentration versus time plots at different $\left[\mathrm{PPh}_{3 \mathrm{~d}}\right]$ were use to feed the parameter estimation mode of COPASI Standard deviations $(\sigma)$ of the fitted rate constants are given by COPASI. Values are expressed with their interval confidence and a coverage factor of 2 (level of confidence $95 \%) ; X_{i} \pm 2 \sigma$.

\section{Transmetalation from the trans isomer}

The following model is proposed for the isomerization of (2a):

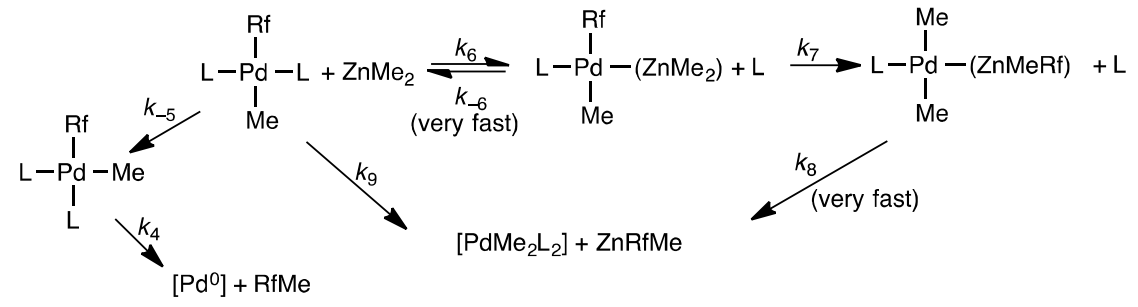

Scheme S2. Proposed model for the transmetalation reaction between trans-[PdRfMe $\left.\left(\mathrm{PPh}_{3}\right)_{2}\right]$ and $\mathrm{ZnMe}_{2}$.

Rate constants $\mathrm{k}_{6}, \mathrm{k}-6_{6} \mathrm{k}_{7}$ and $\mathrm{k}_{9}$ were fitted according to the proposed model of 0 . Constant $\mathrm{k}_{-5}$ was fixed for every experiment at different $\left[\mathrm{PPh}_{3}\right]$, as the experimental value obtained in the cis-trans isomerization modeling $\left(\mathrm{k}_{-5}=2.46 \cdot 10^{-5} \mathrm{~s}^{-1}\right)$. $\mathrm{k}_{8}$ is subsequent to a slow equilibrium and since it is meant to be very fast, no suitable values can be obtained with a data fitting. When the modeling was tested with values over 1000 for $\mathrm{k}_{8}$, the other constants remained unaffected. $\mathrm{k}_{4}$ was fixed according to the literature data $\left(\mathrm{k}_{4}=8.9 \cdot 10^{-6} \mathrm{~s}^{-1}\right){ }^{2}$

The fitting showed a covariance of 0.998 for the pair of variables $\mathrm{k}_{7}$ and $\mathrm{k}_{9}$, meaning that this pair of values cannot be simultaneously calculated with the data available. Independently of $\mathrm{k}_{7}$ and $\mathrm{k}_{9}, \mathrm{k}_{6}$ has a value of $0.135 * \mathrm{k}_{6}$ for $\mathrm{k}_{6}>0.1$, so the substitution of the ligand $\mathrm{L}=\mathrm{PPh}_{3}$ in 
trans- $\left[\mathrm{PdRfMeL}_{2}\right]$ can be kinetically envisioned as a fast preequilibrium with an equilibrium constant of $\mathrm{K}_{6}=0.135$. A maximum value for $\mathrm{k}_{9}$ was estimated assuming that under the maximum concentration of $\left[\mathrm{PPh}_{3}\right]$ used, the transformation through $\mathrm{k}_{6}$ were negligible, obtaining $\mathrm{k}_{9}=2.4 \cdot 10^{-5} \mathrm{~mol}^{-1} \mathrm{~s}^{-1}$. Conversely, assuming this maximum value for $\mathrm{k}_{9}$, a minimum value for $\mathrm{k}_{7}=2.12 \cdot 10^{-5} \mathrm{~s}^{-1}$ was obtained.

Table S5. Fitted rate constants according to the reaction model.

\begin{tabular}{cc} 
& Values $(\mathrm{st} \mathrm{dv})$ \\
\hline $\mathrm{k}_{6}$ & $13.50(0.05)\left(\mathrm{mol}^{-1} \cdot \mathrm{s}^{-1}\right)$ \\
$\mathrm{k}_{-6}$ & $100\left(\mathrm{~s}^{-1}\right)($ fixed $)$ \\
$\mathrm{k}_{7}$ & $2.12(0.01) \cdot 10^{-5}\left(\mathrm{~s}^{-1}\right)$ \\
$\mathrm{k}_{8}$ & $1000\left(\mathrm{~mol}^{-1} \cdot \mathrm{s}^{-1}\right)($ fixed $)$ \\
$\mathrm{k}_{9}$ & $2.49 \cdot 10^{-5}\left(\mathrm{~mol}^{-1} \cdot \mathrm{s}^{-1}\right)($ fixed $)$ \\
$\mathrm{k}_{4}$ & $8.90 \cdot 10^{-6}\left(\mathrm{~s}^{-1}\right)($ fixed $)$ \\
$\mathrm{k}_{-5}$ & $2.46 \cdot 10^{-5}\left(\mathrm{~s}^{-1}\right)($ fixed $)$
\end{tabular}

Transmetalation from the cis isomer

The following model is proposed for the isomerization of (1a):

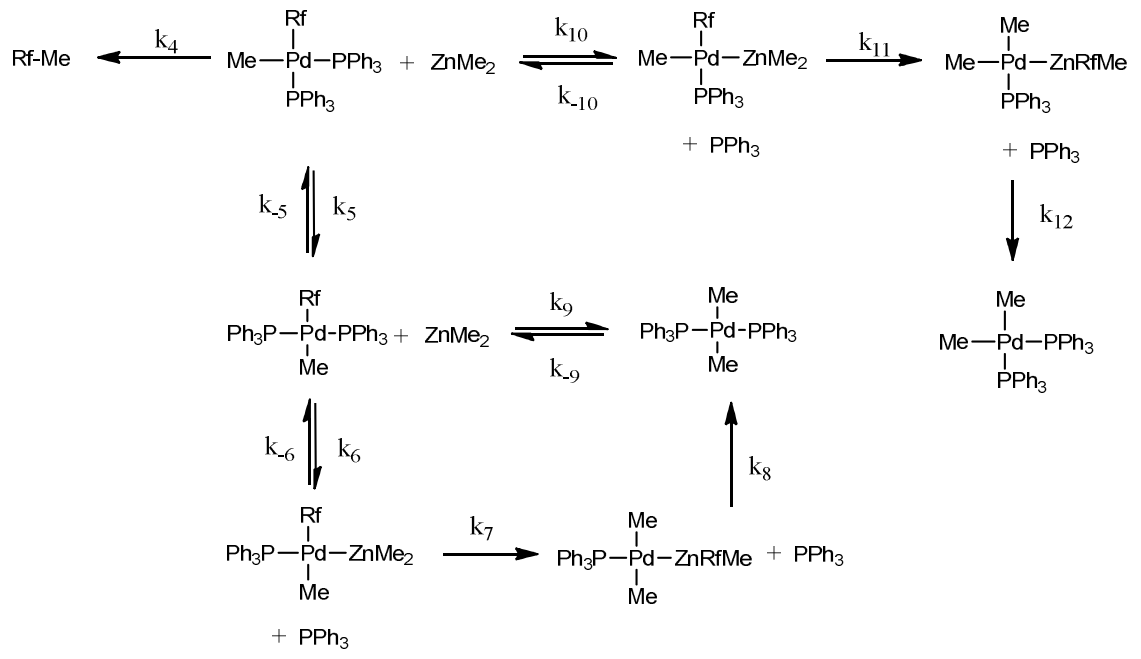

Scheme S3. Proposed model for the transmetalation reaction between cis-[PdRfMe $\left.\left(\mathrm{PPh}_{3}\right)_{2}\right]$ and $\mathrm{ZnMe}_{2}$. 
Table S6. Fitted rate constants according to the reaction model.

\begin{tabular}{cc}
\hline & Values \\
\hline $\mathrm{k}_{10}$ & $(2.5 \pm 0.1) \cdot 10^{-4}\left(\mathrm{~mol}^{-1} \cdot \mathrm{s}^{-1}\right)$ \\
$\mathrm{k}_{-10}$ & $4.2 \pm 0.1\left(\mathrm{~s}^{-1}\right)$ \\
$\mathrm{k}_{11}$ & $(4.5 \pm 1) \cdot 10^{-4}\left(\mathrm{~s}^{-1}\right)$ \\
$\mathrm{k}_{12}$ & $1000\left(\mathrm{~mol}^{-1} \cdot \mathrm{s}^{-1}\right)$ \\
$\mathrm{k}_{4}$ & $8.90 \cdot 10^{-6}\left(\mathrm{~s}^{-1}\right)$ \\
$\mathrm{k}_{5}$ & $4.67 \cdot 10^{-5}\left(\mathrm{~s}^{-1}\right)$ (fixed) \\
$\mathrm{k}_{-5}$ & $2.46 \cdot 10^{-5}\left(\mathrm{~s}^{-1}\right)$ (fixed)
\end{tabular}

Rate constants $\mathrm{k}_{10}, \mathrm{k}_{-10}$ and $\mathrm{k}_{11}$ were fitted according to the proposed model of 0 . Constants $\mathrm{k}_{5}$ and $\mathrm{k}_{-5}$ were fixed for every experiment at different $\left[\mathrm{PPh}_{3}\right]$, as the experimental value obtained in the cis-trans isomerization modeling $\left(\mathrm{k}_{5}=4.67 \cdot 10^{-5} \mathrm{~s}^{-1}, \mathrm{k}_{-5}=2.46 \cdot 10^{-5} \mathrm{~s}^{-1}\right) . \mathrm{k}_{4}$ was fixed according to the literature data $\left(\mathrm{k}_{4}=8.9 \cdot 10^{-6} \mathrm{~s}^{-1}\right)$. Error! Bookmark not defined. $\mathrm{k}_{12}$ is subsequent to a slow equilibrium and since it is meant to be very fast, no suitable values can be obtained with a data fitting. When the modeling was tested with values over 1000 for $\mathrm{k}_{8}$, the other constants remained unaffected. Constants $\mathrm{k}_{6}, \mathrm{k}_{7}, \mathrm{k}_{8}, \mathrm{k}_{9}$ and $\mathrm{k}_{-9}$ were fixed as obtained from the transmetalation from the trans isomer. Since the transmetalation from the trans isomer is faster in the studied range of $\left[\mathrm{PPh}_{3}\right]$, these reactions were included to take into account the $\mathrm{ZnRfMe}$ formation for the trans isomer .

Table S7. Transmetalation of the complex trans-[PdRfCl$\left.\left(\mathrm{PPh}_{3}\right)_{2}\right]$.

\begin{tabular}{lcccc}
\hline$\left[\mathrm{PPh}_{3}\right]$ & $\begin{array}{c}-\mathrm{r}^{0}\left(\mathrm{M} \cdot \mathrm{s}^{-1}\right) \\
\left(\mathrm{t}-\left[\mathrm{PdRfCl}\left(\mathrm{PPh}_{3}\right)_{2}\right]\right)\end{array}$ & $\begin{array}{c}\mathrm{r}^{0}\left(\mathrm{M} \cdot \mathrm{s}^{-1}\right) \\
(\mathrm{ZnRfMe})\end{array}$ & $\begin{array}{c}\mathrm{r}^{0}\left(\mathrm{M} \cdot \mathrm{s}^{-1}\right) \\
\left(\mathrm{t}-\left[\mathrm{PdRfMe}\left(\mathrm{PPh}_{3}\right)_{2}\right]\right)\end{array}$ & $\begin{array}{c}\mathrm{r}^{0}\left(\mathrm{M} \cdot \mathrm{s}^{-1}\right) \\
\left(\mathrm{c}-\left[\mathrm{PdRfMe}\left(\mathrm{PPh}_{3}\right)_{2}\right]\right)\end{array}$ \\
\hline $4.00 \cdot 10^{-04}$ & $6.10 \mathrm{e}-06$ & $3.48 \mathrm{e}-07$ & $5.60 \mathrm{e}-06$ & $1.30 \mathrm{e}-07$ \\
$8.00 \cdot 10^{-04}$ & $2.80 \mathrm{e}-06$ & $2.57 \mathrm{e}-07$ & $3.80 \mathrm{e}-06$ & $9.33 \mathrm{e}-08$ \\
$1.60 \cdot 10^{-03}$ & $1.72 \mathrm{e}-06$ & $2.08 \mathrm{e}-07$ & $1.85 \mathrm{e}-06$ & $9.15 \mathrm{e}-08$ \\
$4.00 \cdot 10^{-03}$ & $1.30 \mathrm{e}-06$ & $1.30 \mathrm{e}-07$ & $1.30 \mathrm{e}-06$ & $6.76 \mathrm{e}-08$ \\
$1.60 \cdot 10^{-02}$ & $1.61 \mathrm{e}-07$ & $3.31 \mathrm{e}-08$ & $5.10 \mathrm{e}-08$ & $7.30 \mathrm{e}-08$
\end{tabular}




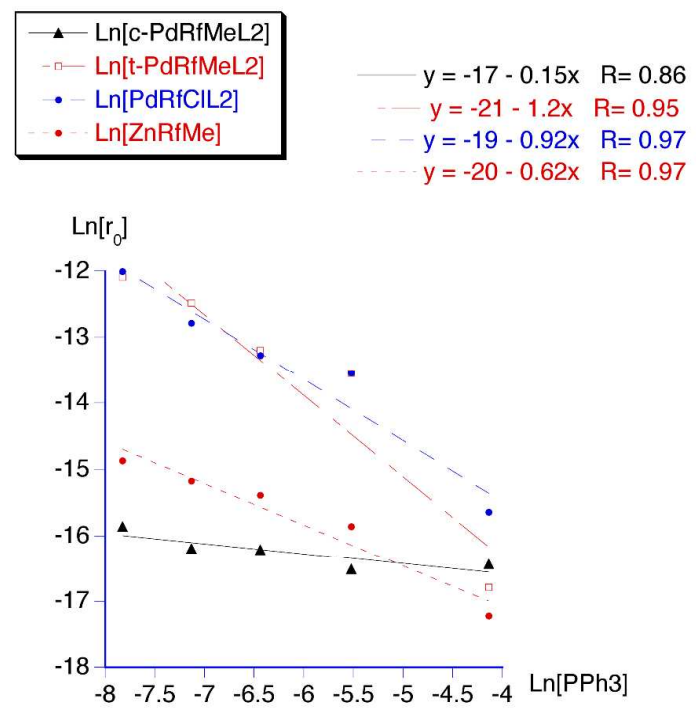

Figure S3. Plot of the logarithmic rate of disappearance of trans-[PdRfCl$\left.\left(\mathrm{PPh}_{3}\right)_{2}\right](\mathbf{1})\left(\operatorname{lnr}{ }^{0}\right.$, black triangles), and of formation of complexes trans-[PdRfMe $\left.\left(\mathrm{PPh}_{3}\right)_{2}\right](2)$ (red spots), cis[PdRfMe $\left.\left(\mathrm{PPh}_{3}\right)_{2}\right]$ (3) (blue spots), and ZnRfMe (green triangles) vs. $\ln \left[\mathrm{PPh}_{3}\right]$. The slopes correspond to the experimental reaction order on $\left[\mathrm{PPh}_{3}\right]$ for these reactions.

The kinetic constants were fitted according to the following model.

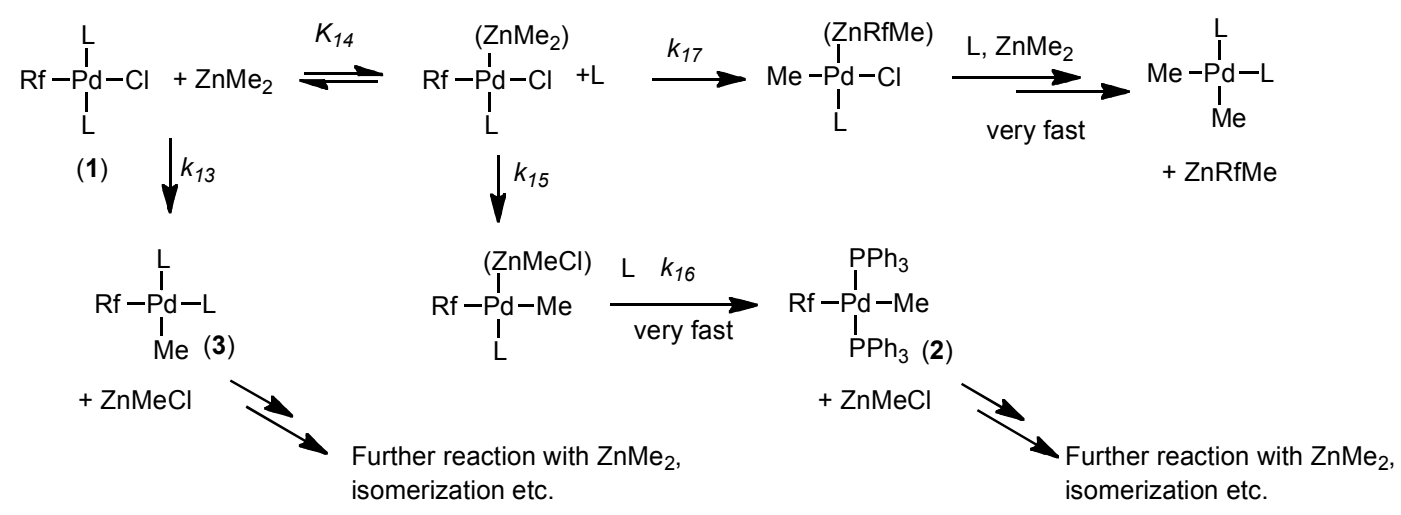

Scheme S4. Proposed model for the transmetalation reaction between trans- $\left[\mathrm{PdRf}\left(\mathrm{PPh}_{3}\right)_{2}\right]$ and $\mathrm{ZnMe}_{2}$.

Fitted rate constants according to the reaction model.

Values

\begin{tabular}{cc} 
& Values \\
\hline $\mathrm{k}_{13}$ & $(3.3 \pm 0.1) \cdot 10^{-4}\left(\mathrm{~mol}^{-1} \cdot \mathrm{s}^{-1}\right)$ \\
$\mathrm{K}_{14}$ & $(9.6 \pm 0.7) \cdot 10^{-3}$ \\
$\mathrm{k}_{15}$ & $(1.1 \pm 0.1) \cdot 10^{-3}\left(\mathrm{~s}^{-1}\right)$ \\
$\mathrm{k}_{17}$ & $(5.2 \pm 0.1) \cdot 10^{-5}\left(\mathrm{~s}^{-1}\right)$
\end{tabular}


Transmetalation from the trans isomer

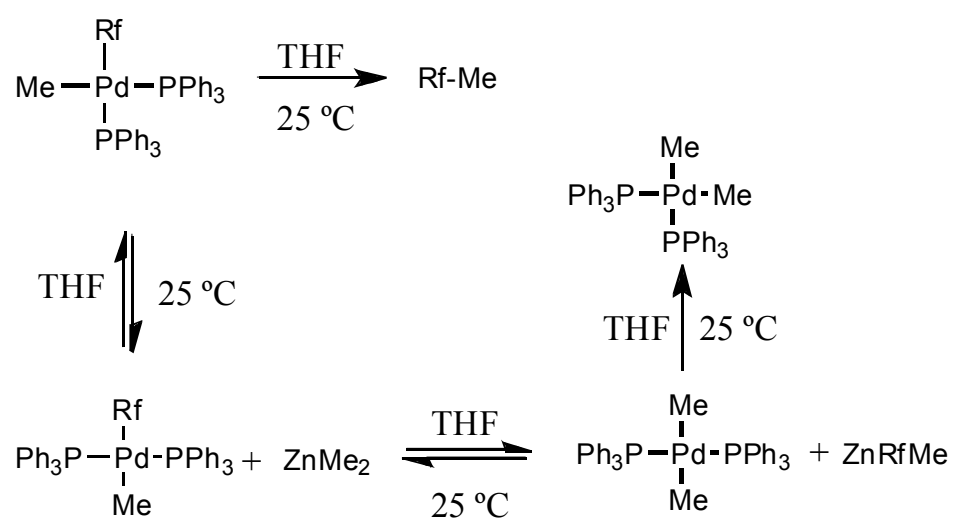

Scheme S5. kinetic model for the transmetalation reaction between trans-[PdRfMe $\left.\left(\mathrm{PPh}_{3}\right)_{2}\right]$ and $\mathrm{ZnMe}_{2}$.

\section{Data input for non-linear least square fitting}

COPASI was fed with concentration versus time data to carry out the fitting of the kinetic constants according with the proposed models. The attached data were obtained by ${ }^{19} \mathrm{~F} \mathrm{NMR}$ integration at $25^{\circ} \mathrm{C}$, only $10 \%$ of the first points of the plots. Time is provided in seconds and concentration data in $\mathrm{M}$.

Table S8. Transmetalation reaction between trans-[PdRfCl$\left.\left(\mathrm{PPh}_{3}\right)_{2}\right]$ and $\mathrm{ZnMe}_{2}$.

$\begin{array}{cccccc}\text { Time } & {\left[\mathrm{PPh}_{3}\right]} & \text { cis-[PdRfMeL2] } & \text { trans-[PdRfMeL } & \text { trans-[PdRfClL } & \text { [PnRfMe } \\ 312 & 0.016000 & 0.00000 & 0.00000 & 0.00800 & 0.00000 \\ 1284 & 0.016000 & 0.00000 & 0.00000 & 0.00800 & 0.00000 \\ 2256 & 0.016000 & 0.00016 & 0.00013 & 0.00771 & 0.00000 \\ 3228 & 0.016000 & 0.00027 & 0.00016 & 0.00749 & 0.00008 \\ 6072 & 0.016000 & 0.00042 & 0.00030 & 0.00708 & 0.00020 \\ 7944 & 0.016000 & 0.00060 & 0.00036 & 0.00674 & 0.00031 \\ 9816 & 0.016000 & 0.00061 & 0.00055 & 0.00650 & 0.00035 \\ 11688 & 0.016000 & 0.00078 & 0.00058 & 0.00626 & 0.00038 \\ 15360 & 0.016000 & 0.00094 & 0.00069 & 0.00590 & 0.00048 \\ 19032 & 0.016000 & 0.00112 & 0.00089 & 0.00546 & 0.00052 \\ 22704 & 0.016000 & 0.00132 & 0.00104 & 0.00508 & 0.00057 \\ 0 & 0.016000 & 0.00000 & 0.00000 & 0.00800 & 0.00000 \\ 510 & 0.0040000 & 0.00000 & 0.00100 & 0.00700 & 0.00000\end{array}$




\begin{tabular}{|c|c|c|c|c|c|}
\hline 780 & 0.0040000 & 0.00008 & 0.00137 & 0.00647 & 0.00008 \\
\hline 1050 & 0.0040000 & 0.00011 & 0.00167 & 0.00611 & 0.00011 \\
\hline 1320 & 0.0040000 & 0.00013 & 0.00192 & 0.00581 & 0.00014 \\
\hline 1590 & 0.0040000 & 0.00014 & 0.00213 & 0.00557 & 0.00016 \\
\hline 2040 & 0.0040000 & 0.00016 & 0.00240 & 0.00517 & 0.00025 \\
\hline 2490 & 0.0040000 & 0.00021 & 0.00269 & 0.00480 & 0.00027 \\
\hline 2940 & 0.0040000 & 0.00024 & 0.00289 & 0.00454 & 0.00034 \\
\hline 3390 & 0.0040000 & 0.00027 & 0.00310 & 0.00421 & 0.00038 \\
\hline 3840 & 0.0040000 & 0.00030 & 0.00329 & 0.00397 & 0.00040 \\
\hline 4590 & 0.0040000 & 0.00034 & 0.00359 & 0.00358 & 0.00043 \\
\hline 5340 & 0.0040000 & 0.00038 & 0.00384 & 0.00325 & 0.00049 \\
\hline 6090 & 0.0040000 & 0.00041 & 0.00410 & 0.00293 & 0.00051 \\
\hline 6840 & 0.0040000 & 0.00045 & 0.00430 & 0.00267 & 0.00053 \\
\hline 7590 & 0.0040000 & 0.00049 & 0.00448 & 0.00244 & 0.00054 \\
\hline 0 & 0.0040000 & 0.00000 & 0.00000 & 0.00800 & 0.00000 \\
\hline 510 & 0.0016000 & 0.00000 & 0.00111 & 0.00680 & 0.00010 \\
\hline 780 & 0.0016000 & 0.00000 & 0.00150 & 0.00632 & 0.00016 \\
\hline 1050 & 0.0016000 & 0.00008 & 0.00182 & 0.00582 & 0.00023 \\
\hline 1320 & 0.0016000 & 0.00009 & 0.00214 & 0.00540 & 0.00031 \\
\hline 1590 & 0.0016000 & 0.00013 & 0.00241 & 0.00501 & 0.00037 \\
\hline 2040 & 0.0016000 & 0.00015 & 0.00287 & 0.00444 & 0.00046 \\
\hline 2490 & 0.0016000 & 0.00020 & 0.00330 & 0.00388 & 0.00051 \\
\hline 2940 & 0.0016000 & 0.00024 & 0.00365 & 0.00338 & 0.00060 \\
\hline 3390 & 0.0016000 & 0.00029 & 0.00406 & 0.00289 & 0.00064 \\
\hline 3840 & 0.0016000 & 0.00030 & 0.00448 & 0.00243 & 0.00065 \\
\hline 0 & 0.0016000 & 0.00000 & 0.00000 & 0.00800 & 0.00000 \\
\hline 450 & 0.00080000 & 0.00000 & 0.00180 & 0.00608 & 0.00012 \\
\hline 720 & 0.00080000 & 0.00000 & 0.00248 & 0.00533 & 0.00019 \\
\hline 990 & 0.00080000 & 0.00008 & 0.00294 & 0.00472 & 0.00026 \\
\hline 1260 & 0.00080000 & 0.00009 & 0.00348 & 0.00411 & 0.00033 \\
\hline 1530 & 0.00080000 & 0.00012 & 0.00379 & 0.00361 & 0.00040 \\
\hline 1980 & 0.00080000 & 0.00013 & 0.00433 & 0.00293 & 0.00051 \\
\hline 2430 & 0.00080000 & 0.00022 & 0.00468 & 0.00234 & 0.00063 \\
\hline 0 & 0.00080000 & 0.00000 & 0.00000 & 0.00800 & 0.00000 \\
\hline 780 & 0.00040000 & 0.00045 & 0.00341 & 0.00185 & 0.00212 \\
\hline 1050 & 0.00040000 & 0.00044 & 0.00344 & 0.00177 & 0.00227 \\
\hline 1320 & 0.00040000 & 0.00047 & 0.00341 & 0.00169 & 0.00231 \\
\hline 1590 & 0.00040000 & 0.00053 & 0.00342 & 0.00163 & 0.00230 \\
\hline 2040 & 0.00040000 & 0.00060 & 0.00350 & 0.00153 & 0.00226 \\
\hline 2490 & 0.00040000 & 0.00063 & 0.00350 & 0.00147 & 0.00229 \\
\hline
\end{tabular}




$\begin{array}{cccccc}2940 & 0.00040000 & 0.00070 & 0.00356 & 0.00136 & 0.00227 \\ 3390 & 0.00040000 & 0.00072 & 0.00359 & 0.00129 & 0.00228 \\ 3840 & 0.00040000 & 0.00078 & 0.00359 & 0.00124 & 0.00226 \\ 4590 & 0.00040000 & 0.00079 & 0.00368 & 0.00111 & 0.00230 \\ 5340 & 0.00040000 & 0.00089 & 0.00378 & 0.00100 & 0.00220 \\ 6090 & 0.00040000 & 0.00091 & 0.00385 & 0.00089 & 0.00221 \\ 6840 & 0.00040000 & 0.00096 & 0.00392 & 0.00082 & 0.00216 \\ 7590 & 0.00040000 & 0.00099 & 0.00403 & 0.00075 & 0.00210 \\ 0 & 0.00040000 & 0.00000 & 0.00000 & 0.00800 & 0.00000 \\ 450 & 0.00040000 & 0.00000 & 0.00228 & 0.00560 & 0.00013 \\ 720 & 0.00040000 & 0.00000 & 0.00358 & 0.00419 & 0.00023 \\ 990 & 0.00040000 & 0.00007 & 0.00530 & 0.00230 & 0.00034\end{array}$

Table S9. Transmetalation reaction between trans-[PdRfMe $\left.\left(\mathrm{PPh}_{3}\right)_{2}\right]$ and $\mathrm{ZnMe}_{2}$.

\begin{tabular}{|c|c|c|c|c|c|c|c|}
\hline \multicolumn{8}{|c|}{$[$ cis- $\quad[$ trans- } \\
\hline Time & {$\left[\mathrm{PPh}_{3}\right]$} & {$\left[\mathrm{ZnMe}_{2}\right]$} & $\mathrm{k}_{-5}$ & $\left.\operatorname{PdRfMe}\left(\mathrm{PPh}_{3}\right)_{2}\right]$ & $\left.\operatorname{PdRfMe}\left(\mathrm{PPh}_{3}\right)_{2}\right]$ & ZnRfMe & {$[\mathrm{Rf}-\mathrm{Me}]$} \\
\hline 448 & 0.01671 & 0.667 & $7.11 \mathrm{E}-07$ & $5.0706 \mathrm{E}-05$ & 0.01964 & $2.5272 \mathrm{E}-05$ & $6.409 \mathrm{E}-06$ \\
\hline 1048 & 0.01671 & 0.667 & $7.11 \mathrm{E}-07$ & $7.4434 \mathrm{E}-05$ & 0.01944 & 0.00018724 & $1.5615 \mathrm{E}-05$ \\
\hline 1648 & 0.01671 & 0.667 & $7.11 \mathrm{E}-07$ & $7.5514 \mathrm{E}-05$ & 0.01927 & 0.00034817 & $2.8097 \mathrm{E}-05$ \\
\hline 2248 & 0.01671 & 0.667 & $7.11 \mathrm{E}-07$ & 7.6813E-05 & 0.01912 & 0.00049565 & $2.3786 \mathrm{E}-05$ \\
\hline 2848 & 0.01671 & 0.667 & $7.11 \mathrm{E}-07$ & $9.5565 \mathrm{E}-05$ & 0.01896 & 0.00063515 & $2.7509 \mathrm{E}-05$ \\
\hline 3448 & 0.01671 & 0.667 & $7.11 \mathrm{E}-07$ & $7.1143 \mathrm{E}-05$ & 0.01884 & 0.00078063 & $2.0761 \mathrm{E}-05$ \\
\hline 4048 & 0.01671 & 0.667 & $7.11 \mathrm{E}-07$ & $9.1855 \mathrm{E}-05$ & 0.01869 & 0.00092155 & $1.741 \mathrm{E}-05$ \\
\hline 4648 & 0.01671 & 0.667 & $7.11 \mathrm{E}-07$ & $8.1297 \mathrm{E}-05$ & 0.01856 & 0.00105166 & $2.7765 \mathrm{E}-05$ \\
\hline 5248 & 0.01671 & 0.667 & $7.11 \mathrm{E}-07$ & $9.1037 \mathrm{E}-05$ & 0.01844 & 0.0011643 & $2.1866 \mathrm{E}-05$ \\
\hline 5848 & 0.01671 & 0.667 & $7.11 \mathrm{E}-07$ & $6.4612 \mathrm{E}-05$ & 0.01833 & 0.00129195 & $3.2863 \mathrm{E}-05$ \\
\hline 6448 & 0.01671 & 0.667 & $7.11 \mathrm{E}-07$ & $8.9575 \mathrm{E}-05$ & 0.0181 & 0.00140983 & $3.4255 \mathrm{E}-05$ \\
\hline 388 & 0.00624 & 0.667 & $1.447 \mathrm{E}-06$ & 0.00016065 & 0.01949 & $3.1661 \mathrm{E}-05$ & $-6.4698 \mathrm{E}-05$ \\
\hline 988 & 0.00624 & 0.667 & $1.447 \mathrm{E}-06$ & 0.00017004 & 0.01920 & 0.00031111 & $-5.6113 \mathrm{E}-05$ \\
\hline 1588 & 0.00624 & 0.667 & $1.447 \mathrm{E}-06$ & 0.00015673 & 0.01893 & 0.00057082 & $-3.6304 \mathrm{E}-05$ \\
\hline 2188 & 0.00624 & 0.667 & $1.447 \mathrm{E}-06$ & 0.00016575 & 0.01870 & 0.00081147 & $-6.0896 \mathrm{E}-05$ \\
\hline 2788 & 0.00624 & 0.667 & $1.447 \mathrm{E}-06$ & 0.00016196 & 0.0184 & 0.00106642 & $-3.9164 \mathrm{E}-05$ \\
\hline 3388 & 0.00624 & 0.667 & $1.447 \mathrm{E}-06$ & 0.00013741 & 0.01824 & 0.00127687 & $-3.303 E-05$ \\
\hline 3988 & 0.00624 & 0.667 & $1.447 \mathrm{E}-06$ & 0.0001417 & 0.01803 & 0.00147709 & $-2.7008 \mathrm{E}-05$ \\
\hline 4588 & 0.00624 & 0.667 & $1.4474 \mathrm{E}-06$ & 0.00014236 & 0.01784 & 0.0016701 & $-2.6998 \mathrm{E}-05$ \\
\hline 5188 & 0.00624 & 0.667 & $1.447 \mathrm{E}-06$ & 0.00012812 & 0.01767 & 0.00184088 & $-1.9346 \mathrm{E}-05$ \\
\hline 5788 & 0.00624 & 0.667 & $1.447 \mathrm{E}-06$ & 0.00012796 & 0.01751 & 0.00200511 & $-1.9494 \mathrm{E}-05$ \\
\hline \multirow[t]{2}{*}{6388} & 0.00624 & 0.667 & $1.447 \mathrm{E}-06$ & 0.00013849 & 0.01736 & 0.0021464 & $-2.6429 \mathrm{E}-05$ \\
\hline & 0.00325 & 0.667 & $3.42 \mathrm{E}-06$ & 0.00017111 & 0.01925 & 0.00016514 & $6.1635 \mathrm{E}-05$ \\
\hline
\end{tabular}




\begin{tabular}{|c|c|c|c|c|c|c|c|}
\hline 1046 & 0.00325 & 0.667 & $3.42 \mathrm{E}-06$ & 0.0001796 & 0.01890 & 0.00050084 & $7.1624 \mathrm{E}-05$ \\
\hline 1646 & 0.00325 & 0.667 & $3.42 \mathrm{E}-06$ & 0.00015918 & 0.01861 & 0.00080915 & $7.0829 \mathrm{E}-05$ \\
\hline 2246 & 0.00325 & 0.667 & $3.42 \mathrm{E}-06$ & 0.00015266 & 0.01832 & 0.00109834 & $8.2495 \mathrm{E}-05$ \\
\hline 2846 & 0.00325 & 0.667 & $3.42 \mathrm{E}-06$ & 0.0001775 & 0.01802 & 0.00136926 & $8.3038 \mathrm{E}-05$ \\
\hline 3446 & 0.00325 & 0.667 & $3.42 \mathrm{E}-06$ & 0.00019934 & 0.01774 & 0.00161092 & $9.7025 \mathrm{E}-05$ \\
\hline 4046 & 0.00325 & 0.667 & $3.42 \mathrm{E}-06$ & 0.00022103 & 0.01748 & 0.0018427 & 0.00010329 \\
\hline 4646 & 0.00325 & 0.667 & $3.42 \mathrm{E}-06$ & 0.00024601 & 0.01725 & 0.00204893 & 0.00010547 \\
\hline 5246 & 0.00325 & 0.667 & $3.42 \mathrm{E}-06$ & 0.00031729 & 0.01699 & 0.00221919 & 0.00012067 \\
\hline 5846 & 0.00325 & 0.667 & $3.42 \mathrm{E}-06$ & 0.00037461 & 0.01668 & 0.0024698 & 0.00012409 \\
\hline 6446 & 0.00325 & 0.667 & $3.42 \mathrm{E}-06$ & 0.00039597 & 0.01637 & 0.00275204 & 0.00013222 \\
\hline 375 & 0.00153 & 0.667 & $1.58 \mathrm{E}-05$ & 0.00020648 & 0.01928 & 0.0001808 & $6.1629 \mathrm{E}-05$ \\
\hline 975 & 0.00153 & 0.667 & $1.58 \mathrm{E}-05$ & 0.00018791 & 0.01888 & 0.00058902 & 7.3437E-05 \\
\hline 1575 & 0.00153 & 0.667 & $1.58 \mathrm{E}-05$ & 0.000179 & 0.0184 & 0.00100917 & 7.5799E-05 \\
\hline 2175 & 0.00153 & 0.667 & $1.58 \mathrm{E}-05$ & 0.00016537 & 0.01811 & 0.00137035 & 8.2684E-05 \\
\hline 2775 & 0.00153 & 0.667 & $1.58 \mathrm{E}-05$ & 0.00017188 & 0.0177 & 0.00175352 & 8.8678E-05 \\
\hline 3375 & 0.00153 & 0.667 & $1.58 \mathrm{E}-05$ & 0.00018361 & 0.01735 & 0.00209455 & 0.00010001 \\
\hline 3975 & 0.00153 & 0.667 & $1.58 \mathrm{E}-05$ & 0.00022242 & 0.01699 & 0.00242162 & $9.7796 \mathrm{E}-05$ \\
\hline 4575 & 0.00153 & 0.667 & $1.58 \mathrm{E}-05$ & 0.00024776 & 0.01664 & 0.00274243 & 0.00010393 \\
\hline 5175 & 0.00153 & 0.667 & $1.58 \mathrm{E}-05$ & 0.00027355 & 0.01630 & 0.00303645 & 0.00011584 \\
\hline 5775 & 0.00153 & 0.667 & $1.58 \mathrm{E}-05$ & 0.00030754 & 0.01599 & 0.00331126 & 0.00012067 \\
\hline 6375 & 0.00153 & 0.667 & $1.58 \mathrm{E}-05$ & 0.00034536 & 0.01567 & 0.00359066 & 0.00012377 \\
\hline 818 & 0.0100 & 0.667 & $1.052 \mathrm{E}-06$ & 0.0001104 & 0.01922 & 0.00024718 & 5.9684E-05 \\
\hline 1418 & 0.0100 & 0.667 & $1.052 \mathrm{E}-06$ & $8.7357 \mathrm{E}-05$ & 0.01900 & 0.00048565 & $6.2701 \mathrm{E}-05$ \\
\hline 2018 & 0.0100 & 0.667 & $1.052 \mathrm{E}-06$ & $8.639 \mathrm{E}-05$ & 0.01878 & 0.00068661 & 8.0369E-05 \\
\hline 2618 & 0.0100 & 0.667 & $1.052 \mathrm{E}-06$ & $8.105 \mathrm{E}-05$ & 0.01857 & 0.00090228 & $8.2431 \mathrm{E}-05$ \\
\hline 3218 & 0.0100 & 0.667 & $1.052 \mathrm{E}-06$ & 0.00010015 & 0.01837 & 0.00108689 & 7.5334E-05 \\
\hline 3818 & 0.0100 & 0.667 & $1.052 \mathrm{E}-06$ & 0.00010487 & 0.01819 & 0.00125044 & $8.8118 \mathrm{E}-05$ \\
\hline 4418 & 0.0100 & 0.667 & $1.052 \mathrm{E}-06$ & 0.00010552 & 0.01802 & 0.00142806 & 7.7349E-05 \\
\hline 5018 & 0.0100 & 0.667 & $1.052 \mathrm{E}-06$ & $8.6066 \mathrm{E}-05$ & 0.01785 & 0.00159696 & $9.7824 \mathrm{E}-05$ \\
\hline 5618 & 0.0100 & 0.667 & $1.052 \mathrm{E}-06$ & 0.0001021 & 0.01770 & 0.00174437 & 8.2894E-05 \\
\hline 6218 & 0.0100 & 0.667 & $1.052 \mathrm{E}-06$ & 0.00015413 & 0.01750 & 0.00187756 & $9.7975 \mathrm{E}-05$ \\
\hline 6818 & 0.0100 & 0.667 & $1.052 \mathrm{E}-06$ & 0.00011224 & 0.01743 & 0.00200969 & $8.3756 \mathrm{E}-05$ \\
\hline
\end{tabular}


Table S10.Transmetalation reaction between cis-[PdRfMe $\left.\left(\mathrm{PPh}_{3}\right)_{2}\right]$ and $\mathrm{ZnMe}_{2}$.

[cis-

\begin{tabular}{|c|c|c|c|c|}
\hline Time & {$\left[\mathrm{PPh}_{3}\right]$} & {$\left[\mathrm{ZnMe}_{2}\right]$} & $\mathrm{k}_{5}$ & $\left.\operatorname{PdRfMe}\left(\mathrm{PPh}_{3}\right)_{2}\right]$ \\
\hline 803 & 0.01618438 & 0.667 & $1.40 \mathrm{E}-06$ & 0.01946585 \\
\hline 1403 & 0.01618438 & 0.667 & $1.40 \mathrm{E}-06$ & 0.01934087 \\
\hline 003 & 0.01618438 & 0.667 & $1.40 \mathrm{E}-06$ & 0.01918547 \\
\hline 603 & 0.01618438 & 0.667 & $1.40 \mathrm{E}-06$ & 0.01905093 \\
\hline 203 & 0.01618438 & 0.667 & $1.40 \mathrm{E}-06$ & 0.0189342 \\
\hline 303 & 0.01618438 & 0.667 & $1.40 \mathrm{E}-06$ & 0.01878355 \\
\hline 403 & 0.01618438 & 0.667 & $1.40 \mathrm{E}-06$ & 0.01864005 \\
\hline 03 & 0.01618438 & 0.667 & $1.40 \mathrm{E}-06$ & 0.01849763 \\
\hline 603 & 0.01618438 & 0.667 & $1.40 \mathrm{E}-06$ & 0.01836404 \\
\hline 03 & 0.01618438 & 0.667 & $1.40 \mathrm{E}-06$ & 0.01824773 \\
\hline 1014 & 0.01306251 & 0.667 & $1.75 \mathrm{E}-06$ & 0.01921163 \\
\hline 1614 & 0.01306251 & 0.667 & $1.75 \mathrm{E}-06$ & 0.01900929 \\
\hline 214 & 0.01306251 & 0.667 & $1.75 \mathrm{E}-06$ & 0.01897162 \\
\hline 814 & 0.01306251 & 0.667 & $1.75 \mathrm{E}-06$ & 0.01877783 \\
\hline 14 & 0.01306251 & 0.667 & $1.75 \mathrm{E}-06$ & 0.0186487 \\
\hline 014 & 0.01306251 & 0.667 & $1.75 \mathrm{E}-06$ & 0.01852189 \\
\hline 4614 & 0.01306251 & 0.667 & $1.75 \mathrm{E}-06$ & 0.01837151 \\
\hline 5214 & 0.01306251 & 0.667 & $1.75 \mathrm{E}-06$ & 0.01819135 \\
\hline 5814 & 0.01306251 & 0.667 & $1.75 \mathrm{E}-06$ & 0.01804502 \\
\hline 6414 & 0.01306251 & 0.667 & $1.75 \mathrm{E}-06$ & 0.01794278 \\
\hline 683 & 0.00733285 & 0.667 & $2.30 \mathrm{E}-06$ & 0.01957735 \\
\hline 947 & 0.00733285 & 0.667 & $2.30 \mathrm{E}-06$ & 0.0194863 \\
\hline 1547 & 0.00733285 & 0.667 & $2.30 \mathrm{E}-06$ & 0.01942667 \\
\hline 2147 & 0.00733285 & 0.667 & $2.30 \mathrm{E}-06$ & 0.01923104 \\
\hline 2747 & 0.00733285 & 0.667 & $2.30 \mathrm{E}-06$ & 0.01904056 \\
\hline 3347 & 0.00733285 & 0.667 & $2.30 \mathrm{E}-06$ & 0.01887415 \\
\hline 3947 & 0.00733285 & 0.667 & $2.30 \mathrm{E}-06$ & 0.01878456 \\
\hline 4547 & 0.00733285 & 0.667 & $2.30 \mathrm{E}-06$ & 0.01854053 \\
\hline 5147 & 0.00733285 & 0.667 & $2.30 \mathrm{E}-06$ & 0.01839314 \\
\hline 5747 & 0.00733285 & 0.667 & $2.30 \mathrm{E}-06$ & 0.01826545 \\
\hline 6347 & 0.00733285 & 0.667 & $2.30 \mathrm{E}-06$ & 0.01811401 \\
\hline 475 & 0.00478478 & 0.667 & $3.50 \mathrm{E}-06$ & 0.01946284 \\
\hline 747 & 0.00478478 & 0.667 & $3.50 \mathrm{E}-06$ & 0.01939654 \\
\hline 1347 & 0.00478478 & 0.667 & $3.50 \mathrm{E}-06$ & 0.01921687 \\
\hline 1947 & 0.00478478 & 0.667 & $3.50 \mathrm{E}-06$ & 0.01901191 \\
\hline 47 & 0.00478478 & 0.667 & $3.50 \mathrm{E}-06$ & 0.01884883 \\
\hline & 0.00478478 & 0.667 & $3.50 \mathrm{E}-06$ & 0.01863858 \\
\hline
\end{tabular}

[trans-

\begin{tabular}{|c|c|c|}
\hline $\left.\mathrm{dRfMe}\left(\mathrm{PPh}_{3}\right)_{2}\right]$ & ZnRfMe & [Rf-Me] \\
\hline $6.625 \mathrm{E}-05$ & $-1.6921 \mathrm{E}-05$ & 0.00015409 \\
\hline $6.9382 \mathrm{E}-05$ & 4.9935E-06 & 0.00025402 \\
\hline 8.5803E-05 & $2.5869 \mathrm{E}-05$ & 0.00037212 \\
\hline 8.4489E-05 & 4.8969E-05 & 0.00048488 \\
\hline $6.7522 \mathrm{E}-05$ & $6.9481 \mathrm{E}-05$ & 0.00059806 \\
\hline $7.6806 \mathrm{E}-05$ & $9.2843 \mathrm{E}-05$ & 0.00071606 \\
\hline $9.6782 \mathrm{E}-05$ & 0.00011302 & 0.00081941 \\
\hline 0.00011237 & 0.00013346 & 0.0009258 \\
\hline $9.779 \mathrm{E}-05$ & 0.00016188 & 0.00104555 \\
\hline $9.2699 \mathrm{E}-05$ & 0.00017562 & 0.00115321 \\
\hline 0.00015544 & $-1.6245 \mathrm{E}-05$ & 0.00031253 \\
\hline 0.0002236 & $1.0515 \mathrm{E}-05$ & 0.00041995 \\
\hline 0.00012153 & $2.9157 \mathrm{E}-05$ & 0.00054105 \\
\hline 0.00017478 & $6.0754 \mathrm{E}-05$ & 0.00064999 \\
\hline 0.00017822 & 7.9007E-05 & 0.00075742 \\
\hline 0.00015768 & 0.00011417 & 0.00086961 \\
\hline 0.00017016 & 0.00014309 & 0.00097859 \\
\hline 0.0002122 & 0.00017149 & 0.00108832 \\
\hline 0.00021171 & 0.00020338 & 0.00120324 \\
\hline 0.0001802 & 0.000229 & 0.00131137 \\
\hline $3.8571 \mathrm{E}-07$ & $-3.7408 \mathrm{E}-05$ & 0.00014883 \\
\hline $8.7447 \mathrm{E}-05$ & $-3.2187 \mathrm{E}-05$ & 0.0001476 \\
\hline $9.7649 \mathrm{E}-05$ & $-1.3249 \mathrm{E}-05$ & 0.00017809 \\
\hline 0.00012897 & $2.9676 \mathrm{E}-05$ & 0.00029948 \\
\hline 0.00017316 & $6.7521 \mathrm{E}-05$ & 0.00040792 \\
\hline 0.00018781 & 0.00010434 & 0.00052286 \\
\hline 0.00012086 & 0.00013988 & 0.00064386 \\
\hline 0.00021032 & 0.00018366 & 0.00075464 \\
\hline 0.00021645 & 0.00021533 & 0.00086424 \\
\hline 0.00019013 & 0.00026051 & 0.00097307 \\
\hline 0.00017672 & 0.00031212 & 0.00108631 \\
\hline 7.9053E-06 & $-3.8861 \mathrm{E}-05$ & 6.9918E-05 \\
\hline 4.6697E-05 & $-3.9499 \mathrm{E}-05$ & 9.8064E-05 \\
\hline $5.0853 \mathrm{E}-05$ & $1.389 \mathrm{E}-05$ & 0.0002202 \\
\hline $6.8625 \mathrm{E}-05$ & $7.6375 \mathrm{E}-05$ & 0.0003449 \\
\hline $6.689 \mathrm{E}-05$ & 0.00012749 & 0.0004586 \\
\hline $9.3576 \mathrm{E}-05$ & 0.00019025 & 0.0005794 \\
\hline
\end{tabular}




$\begin{array}{cccccccc}3747 & 0.00478478 & 0.667 & 3.50 \mathrm{E}-06 & 0.01847362 & 9.5415 \mathrm{E}-05 & 0.00022985 & 0.00070293 \\ 4347 & 0.00478478 & 0.667 & 3.50 \mathrm{E}-06 & 0.01830291 & 9.0426 \mathrm{E}-05 & 0.00030371 & 0.00080476 \\ 4947 & 0.00478478 & 0.667 & 3.50 \mathrm{E}-06 & 0.01813805 & 7.9726 \mathrm{E}-05 & 0.00035393 & 0.0009301 \\ 5547 & 0.00478478 & 0.667 & 3.50 \mathrm{E}-06 & 0.01790184 & 0.00012248 & 0.00043114 & 0.00104635 \\ 6147 & 0.00478478 & 0.667 & 3.50 \mathrm{E}-06 & 0.01769718 & 0.00015043 & 0.00049762 & 0.00115658 \\ 6747 & 0.00478478 & 0.667 & 3.50 \mathrm{E}-06 & 0.01748657 & 0.0001698 & 0.00056935 & 0.0012761 \\ 485 & 0.00333537 & 0.667 & 6.50 \mathrm{E}-06 & 0.0196832 & 8.5375 \mathrm{E}-05 & -2.8902 \mathrm{E}-05 & 2.8092 \mathrm{E}-05 \\ 1130 & 0.00333537 & 0.667 & 6.50 \mathrm{E}-06 & 0.01934918 & 0.00013087 & 9.6603 \mathrm{E}-05 & 0.00019111 \\ 1420 & 0.00333537 & 0.667 & 6.50 \mathrm{E}-06 & 0.01924537 & 0.00018947 & 0.00012248 & 0.00021045 \\ 2020 & 0.00333537 & 0.667 & 6.50 \mathrm{E}-06 & 0.01905136 & 0.00016025 & 0.00022575 & 0.00033041 \\ 2620 & 0.00333537 & 0.667 & 6.50 \mathrm{E}-06 & 0.01877033 & 0.00023745 & 0.00031992 & 0.00044006 \\ 3220 & 0.00333537 & 0.667 & 6.50 \mathrm{E}-06 & 0.01854224 & 0.00025395 & 0.00041967 & 0.00055191 \\ 3820 & 0.00333537 & 0.667 & 6.50 \mathrm{E}-06 & 0.01836117 & 0.00020703 & 0.00053374 & 0.00066583 \\ 4420 & 0.00333537 & 0.667 & 6.50 \mathrm{E}-06 & 0.01809172 & 0.00023584 & 0.00066972 & 0.00077049 \\ 5020 & 0.00333537 & 0.667 & 6.50 \mathrm{E}-06 & 0.01779768 & 0.00028381 & 0.00081136 & 0.00087492 \\ 278 & 0.0013757 & 0.667 & 4.50 \mathrm{E}-05 & 0.01942258 & 8.965 \mathrm{E}-05 & 8.7949 \mathrm{E}-06 & 7.6335 \mathrm{E}-05 \\ 538 & 0.0013757 & 0.667 & 4.50 \mathrm{E}-05 & 0.01910881 & 0.00029753 & 7.8144 \mathrm{E}-05 & 0.00011288 \\ 1138 & 0.0013757 & 0.667 & 4.50 \mathrm{E}-05 & 0.01885318 & 0.00028858 & 0.00022956 & 0.00022604 \\ 1738 & 0.0013757 & 0.667 & 4.50 \mathrm{E}-05 & 0.01857673 & 0.000301 & 0.00038914 & 0.00033049 \\ 2338 & 0.0013757 & 0.667 & 4.50 \mathrm{E}-05 & 0.01823662 & 0.00032876 & 0.00059563 & 0.00043635\end{array}$




\section{Computational methods}

DFT calculations with the hybrid functionals B3LYP ${ }^{5}$ have been carried out using Gaussian $09^{6}$ to locate and characterize the stationary points on the potential energy surface at the $6-31 \mathrm{G}^{*}$ basis set was used for all atoms with the exception of palladium, for which the Stuttgart-Dresden effective core potential ${ }^{7}$ and the corresponding basis set were used. The optimized geometries have been characterized by harmonic analysis, and the nature of the stationary points determined according to the number of negative eigenvalues of the Hessian matrix. Iinternal reaction coordinates (IRCs) have been followed from the transition structures to verify the proper connections with reactants and products. ${ }^{8}$ Zero-point vibration energies (ZPVE) and thermal corrections (at $298 \mathrm{~K}, 1 \mathrm{~atm}$ ) to the energy have been estimated using the computed frequencies applying the free particle, harmonic oscillator and rigid rotor approximations at the high temperature limit in a canonical ensemble. Solvation effects (to simulate the experimentally used THF) have been also included as single point corrections to the gas-phase free energy of the optimized structures computed with the wB97XD functional ${ }^{9}$ (with dispersion correction) with the self-consistent reaction field (SCRF) method using the polarizable continuum model (PCM) ${ }^{10}$ as implemented in Gaussian09 
Structures of the calculated complexes and transition states

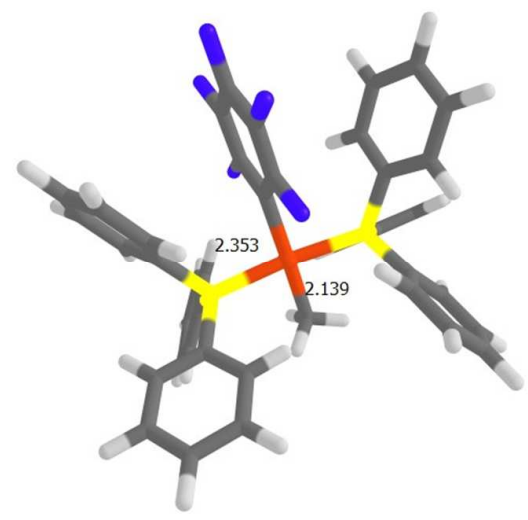

complex2 PPh3

SCF Energy: -2968.23606587581

Num. Imaginary Frequencies: 0

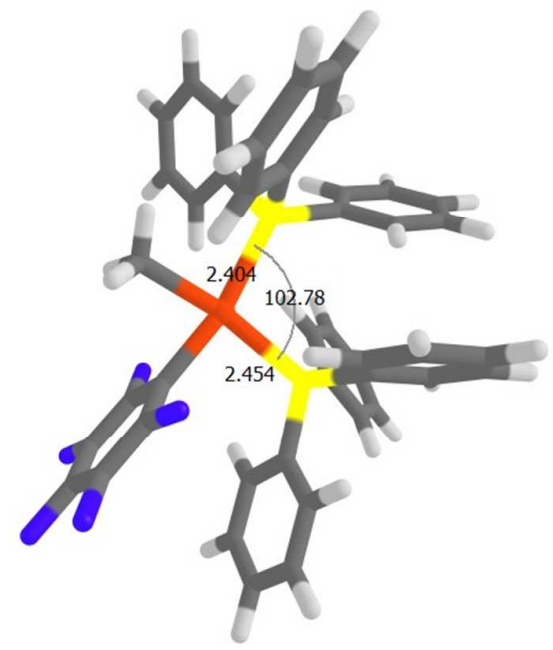

complex3_PPh3

SCF Energy: -2968.233103623161

Num. Imaginary Frequencies: 0

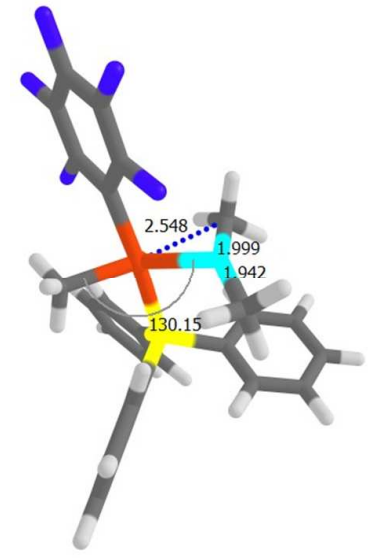

I1_PPh3

SCF Energy: -3790.884764146952

Num. Imaginary Frequencies: 0

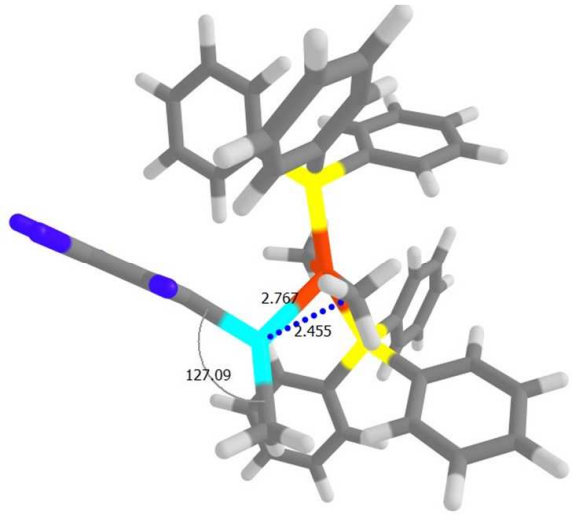

I2_PPh3

SCF Energy: -4827.17280419605

Num. Imaginary Frequencies: 0

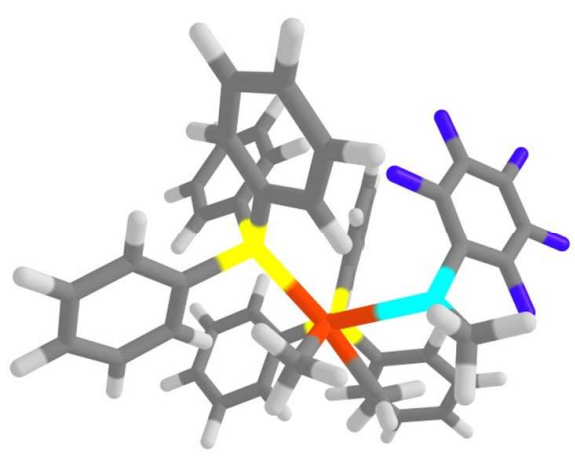

I3_PPh3

SCF Energy: -3676.823382838969

Num. Imaginary Frequencies: 0

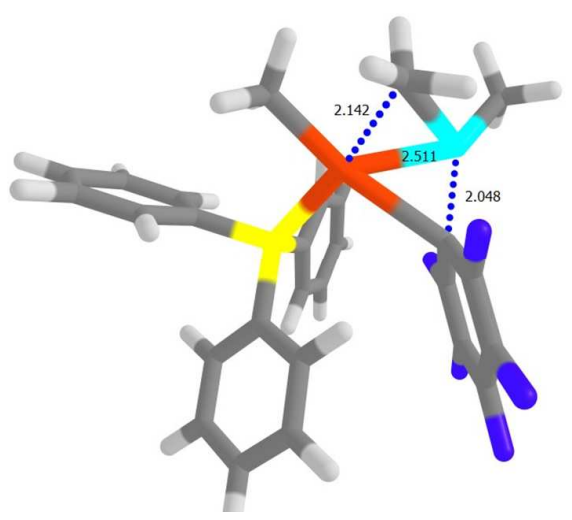

I4_PPh3

SCF Energy: -3790.878514432286

Num. Imaginary Frequencies: 0 


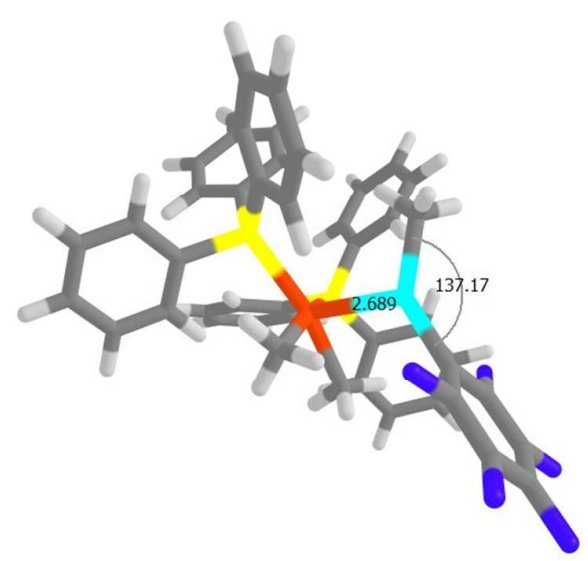

I5_PPh3

SCF Energy: -4827.17560605753

Num. Imaginary Frequencies: 0

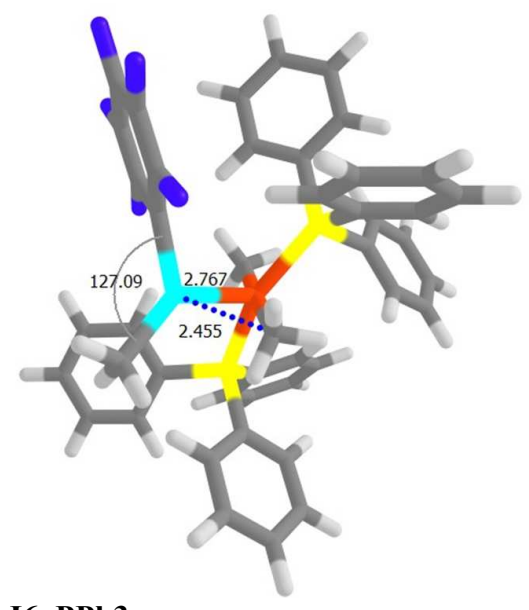

I6 PPh3

SCF Energy: -4827.17280419605

Num. Imaginary Frequencies:0

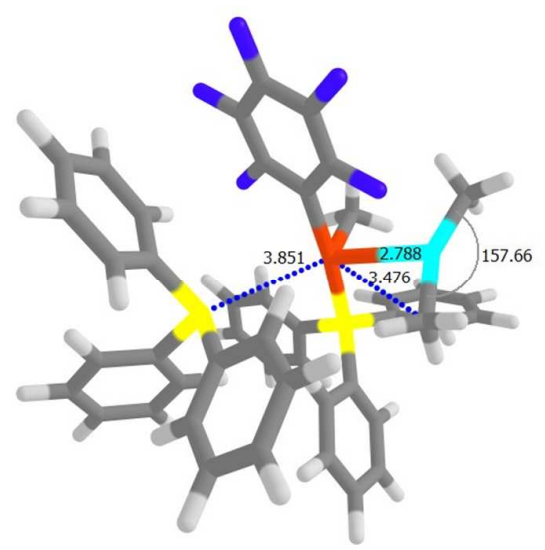

TSI1 PPh3

SCF Energy: -4827.161784119366

Num. Imaginary Frequencies: 1

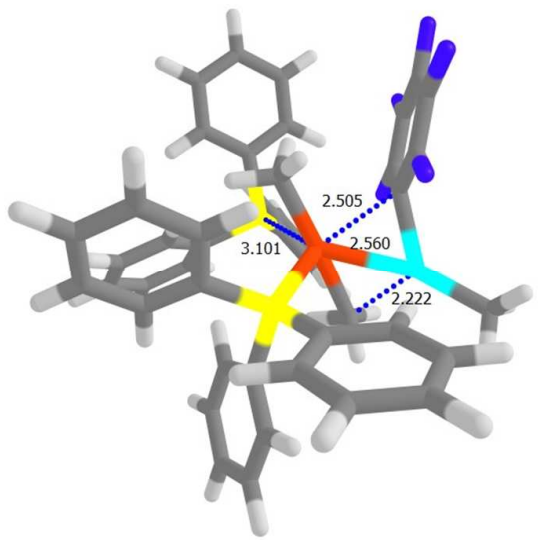

\section{TSI2 PPh3}

SCF Energy: -4827.154762005372

Num. Imaginary Frequencies: 1

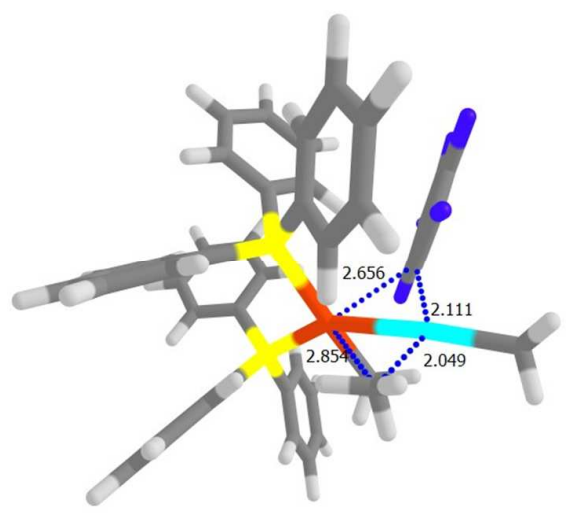

TSI3_PPh3

SCF Energy: -4827.137021744732

Num. Imaginary Frequencies: 1

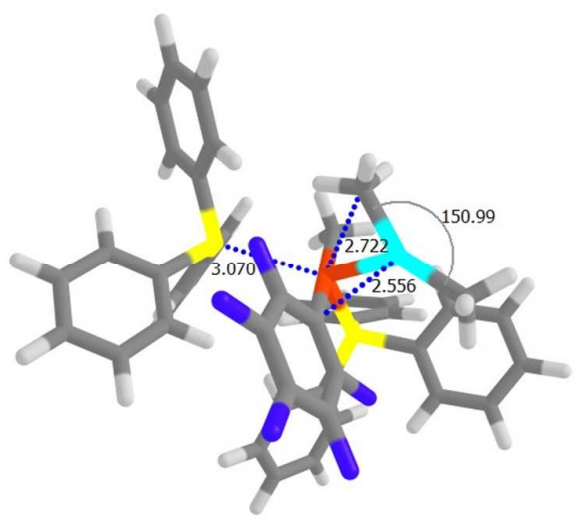

TSI4 PPh3

SCF Energy: -4827.148048350398

Num. Imaginary Frequencies: 1 


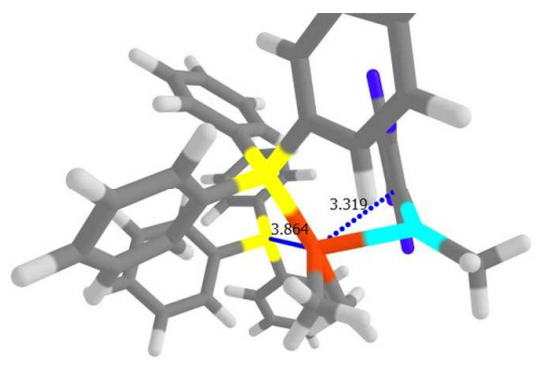

TSI5_PPh3

SCF Ēnergy: -4827.167846044078

Num. Imaginary Frequencies: 1

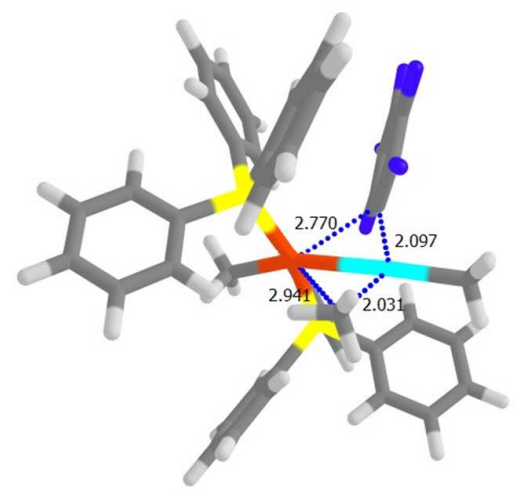

TSI6 PPh3

SCF Energy: -4827.144952319716

Num. Imaginary Frequencies: 1

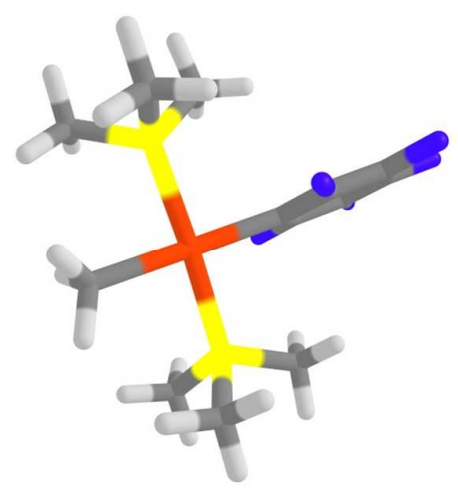

complex2_PMe3

SCF Energy: -1817.873864049881

Num. Imaginary Frequencies: 0

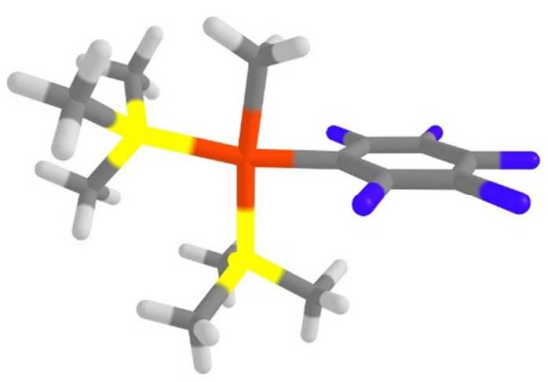

complex3_PMe3

SCF Energy: -1817.866858475873

Num. Imaginary Frequencies: 0

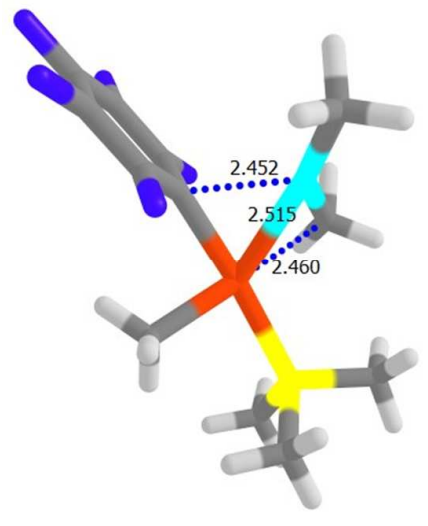

I1_PMe3

SCF Energy: -3215.69513031

Num. Imaginary Frequencies: 0

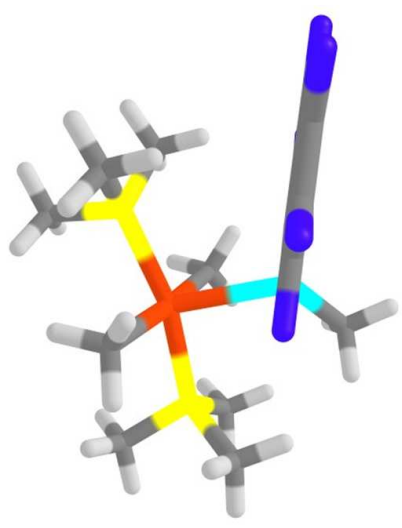

I2 PMe3

SCF Energy: -3215.69513031

Num. Imaginary Frequencies: 0 


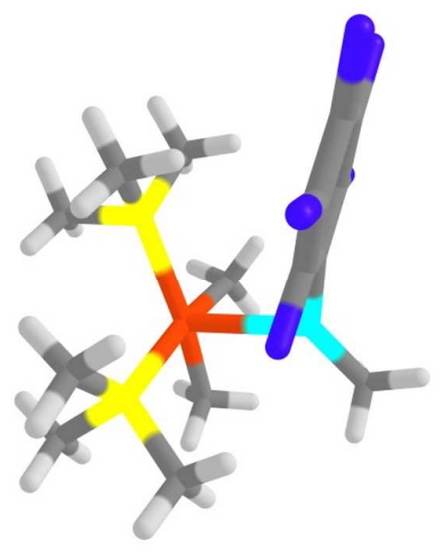

I3 PMe3

SCF Energy: -3676.82108614

Num. Imaginary Frequencies: 0

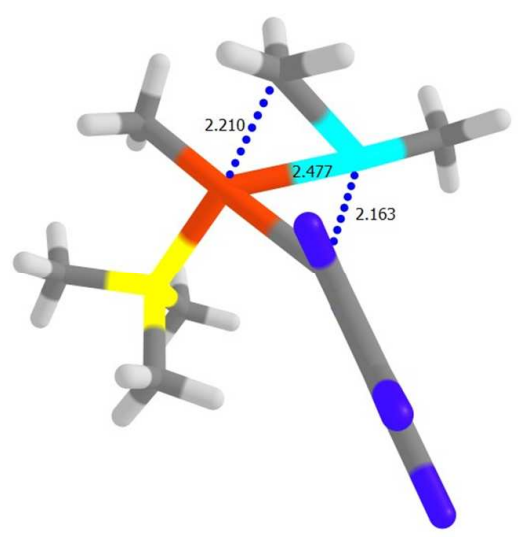

I4_PMe3

SCF Energy: -3676.82108614

Num. Imaginary Frequencies: 0

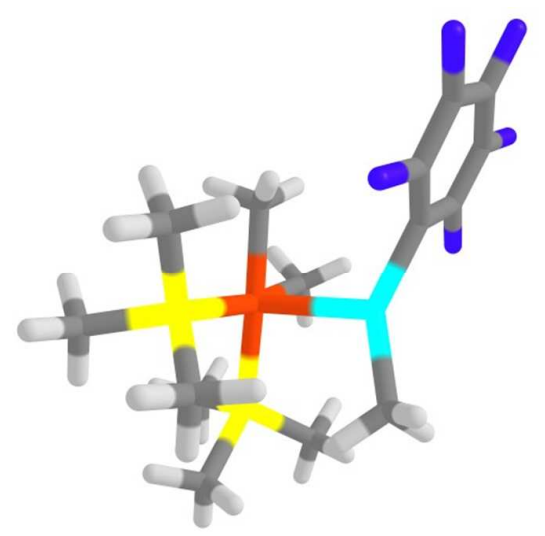

I5 PMe3

SCF Energy: -3676.82108614

Num. Imaginary Frequencies: 0

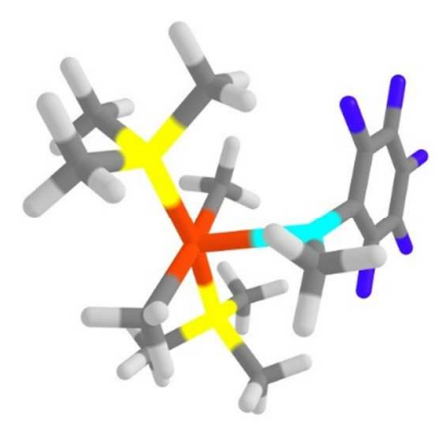

I6_PMe3

SCF Energy: -3676.81625173

Num. Imaginary Frequencies: 0

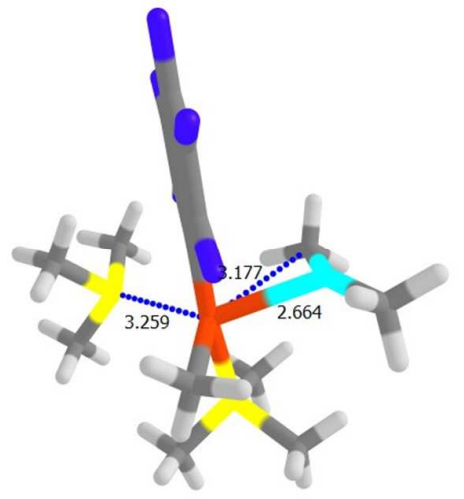

TSI1_PMe3

SCF Energy: -3676.81625173

Num. Imaginary Frequencies: 1

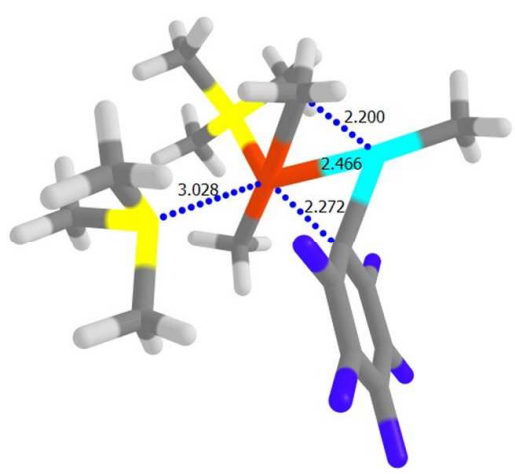

TSI2_PMe3

SCF Ënergy: -3676.78736990

Num. Imaginary Frequencies: 1 


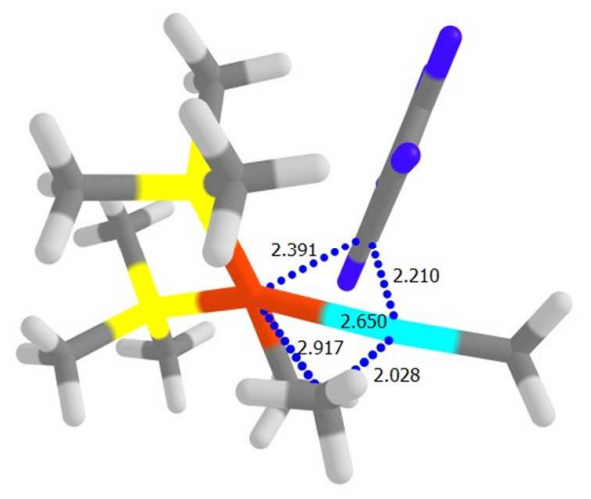

TSI3 PMe3

SCF Ēnergy: -3676.77996043

Num. Imaginary Frequencies: 1

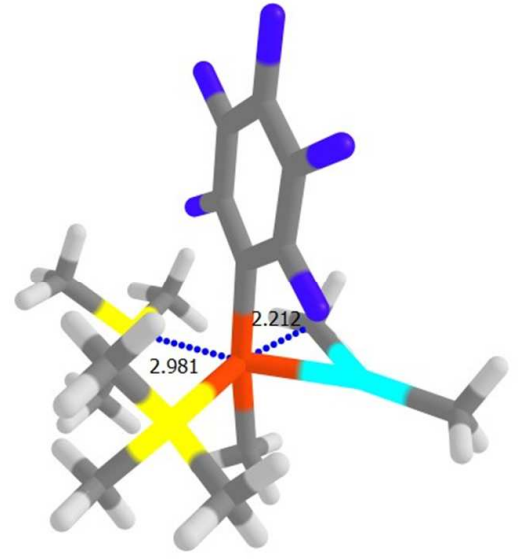

TSI4 PMe3

SCF Energy: -3676.77996043

Num. Imaginary Frequencies: 1

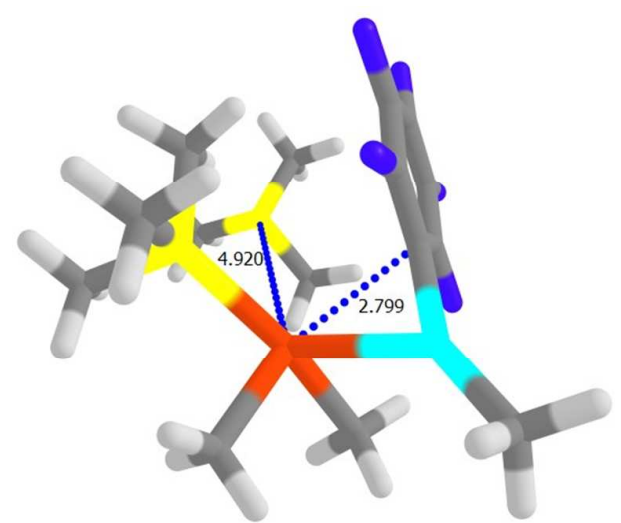

TSI5_PMe3

SCF Energy: -3676.77996043

Num. Imaginary Frequencies: 1

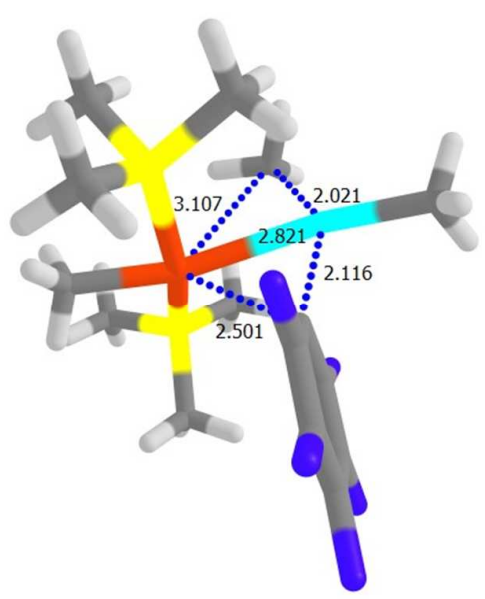

TSI6_PMe3

SCF Energy: -3676.79098824

Num. Imaginary Frequencies: 1 
References:

1. Amman, C.; Meier, P.; Merbach, A. E. J. Magn. Reson. 1982, 46, 319-321.

2. Casares, J. A.; Espinet, P.; Fuentes, B.; Salas, G. J. Am. Chem. Soc., 2007, 129, 35083509 .

3. Gillie A.; Stille J. K. J. Am. Chem. Soc. 1980, 102, 4933-4941.

4. COmplex PAthway SImulator. Hoops, S.; Sahle, S.; Gauges, R.; Lee, C.; Pahle, J.;

Simus, N.; Singhal, M.; Xu, L.; Mendes, P.; Kummer, U. Bioinformatics 2006, 22, 3067-3074.

5 (a) Becke, A. D. J. Chem. Phys. 1993, 98, 5648-5652. (b) Lee, C.; Yang, W.; Parr, R. G. Phys. Rev. B Condens. Matter 1988, 37, 785-789.

6 Frisch, M. J.; Trucks, G. W.; Schlegel, H. B.; Scuseria, G. E.; Robb, M. A.; Cheeseman, J. R.; Scalmani, G.; Barone, V.; Mennucci, B.; Petersson, G. A.; Nakatsuji, H.; Caricato, M.; Li, X.; Hratchian, H. P.; Izmaylov, A. F.; Bloino, J.; Zheng, G.; Sonnenberg, J. L.; Hada, M.; Ehara, M.; Toyota, K.; Fukuda, R.; Hasegawa, J.; Ishida, M.; Nakajima, T.; Honda, Y.; Kitao, O.; Nakai, H.; Vreven, T.; Montgomery, J. A.; Peralta, J. E.; Ogliaro, F.; Bearpark, M.; Heyd, J. J.; Brothers, E.; Kudin, K. N.; Staroverov, V. N.; Kobayashi, R.; Normand, J.; Raghavachari, K.; Rendell, A.; Burant, J. C.; Iyengar, S. S.; Tomasi, J.; Cossi, M.; Rega, N.; Millam, J. M.; Klene, M.; Knox, J. E.; Cross, J. B.; Bakken, V.; Adamo, C.; Jaramillo, J.; Gomperts, R.; Stratmann, R. E.; Yazyev, O.; Austin, A. J.; Cammi, R.; Pomelli, C.; Ochterski, J. W.; Martin, R. L.; Morokuma, K.; Zakrzewski, V. G.; Voth, G. A.; Salvador, P.; Dannenberg, J. J.; Dapprich, S.; Daniels, A. D.; Farkas; Foresman, J. B.; Ortiz, J. V.; Cioslowski, J.; Fox, D. J., Gaussian 09, Revision B.01, Wallingford CT, 2009.

7 Andrae, D.; Häussermann, U.; Dolg, M.; Stoll, H.; Preuss, H. Theor. Chim. Acta. 1990, 77, 123-141.

8 (a) Fukui, K. Acc. Chem. Res. 1981, 14, 363-368. (b) Gonzalez, C.; Schlegel, H. B. J. Phys. Chem. 1990, 94, 5523-5527.

9 Chai, J.-D.; Head-Gordon, M. Phys. Chem. Chem. Phys. 2008, 10, 6615-6620. 
10 (a) Tomasi, J.; Persico, M. Chem. Rev. 1994, 94, 2027-2094. (b) Cossi, M.; Scalmani, G.; Rega, N.; Barone, V. J. Chem. Phys. 2002, 117, 43-54. (c) Tomasi, J.; Mennucci, B.; Cammi, R. Chem. Rev. 2005, 105, 2999-3094. 